\title{
Effectiveness of community physiotherapy and enhanced pharmacy review for knee pain in people aged over 55 presenting to primary care: pragmatic randomised trial
}

Elaine M Hay, Nadine E Foster, Elaine Thomas, George Peat, Mike Phelan, Hannah E Yates, Alison Blenkinsopp, Julius Sim

\begin{abstract}
Objectives To evaluate the effectiveness of two primary care strategies for delivering evidence based care to people aged 55 or over with knee pain: enhanced pharmacy review and community physiotherapy.

Design Pragmatic multicentre randomised clinical trial. Setting 15 general practices in North Staffordshire.

Participants 325 adults aged 55 years or over (mean 68 years) consulting with knee pain; 297 (91\%) reached six month follow-up.

Interventions Enhanced pharmacy review (pharmacological management in accordance with an algorithm); community physiotherapy (advice about activity and pacing and an individualised exercise programme); control (advice leaflet reinforced by telephone call).

Main outcome measure Change in Western Ontario and McMaster Universities osteoarthritis index (WOMAC) at 3, 6, and 12 months.

Results Mean baseline WOMAC pain score was 9.1 (SD 3.7), and mean baseline function score was 29.9 (SD 12.8). At three months, the mean reductions in pain scores were 0.41 (SD 2.8) for control, 1.59 (3.2) for pharmacy, and 1.56 (3.4) for physiotherapy; reductions in function scores were 0.80 (8.5), 2.61 (9.8), and 4.79 (10.8). Compared with control, mean differences in change scores for physiotherapy were $1.15(95 \%$ confidence interval 0.2 to 2.1) for pain and 3.99 (1.2 to 6.8) for function; those for pharmacy were 1.18 (0.3 to 2.1) for pain and 1.80 ( -0.8 to 4.5$)$ for function. These differences were not sustained to six or 12 months. Significantly fewer participants in the physiotherapy group reported consulting their general practitioner for knee pain in the follow-up period, and use of non-steroidal anti-inflammatory drugs was lower in the physiotherapy and pharmacy groups than in the control group.

Conclusions Evidence based care for older adults with knee pain, delivered by primary care physiotherapists and pharmacists, resulted in short term improvements in health outcomes, reduced use of non-steroidal anti-inflammatory drugs, and high patient satisfaction. Physiotherapy seemed to produce a shift in consultation behaviour away from the traditional general practitioner led model of care.

Trial registration UK National Research Register N0286046917; Current Controlled Trials ISRCTN55376150.
\end{abstract}

\section{Introduction}

Current evidence for the primary care management of knee pain and osteoarthritis supports the use of both pharmacological and non-pharmacological approaches. ${ }^{1-5}$ However, the traditional general practitioner led service to deliver such interventions is increasingly unsustainable, and alternative models, using the skills of other members of the primary healthcare team, have been proposed. ${ }^{6}$ For older people with knee pain, at least two services have the potential to provide systematic, effective care. Firstly, an enhanced pharmacy review service by community pharmacists could optimise the drug management of knee pain and provide simple self help messages. Secondly, a community physiotherapy service, which promotes self management alongside an exercise based treatment package, might be a practical way of maximising the benefit of non-drug approaches. To date, the value of each of these services in implementing evidence based care packages for patients with knee pain has not been established. We therefore carried out a pragmatic randomised clinical trial to compare the clinical effectiveness, in primary care, of enhanced pharmacy review or community physiotherapy with that of a control intervention (advice leaflet reinforced by a telephone call) in the treatment of adults aged 55 years and over consulting their general practitioner with knee pain.

\section{Methods}

\section{Study participants}

We recruited participants from 15 general practices in North Staffordshire between May 2001 and March 2004. All adults aged 55 years and over who consulted their general practitioner with pain, stiffness, or both in one or both knees and who were able to give written, informed consent were invited to participate. Exclusion criteria were potentially serious pathology (such as inflammatory arthritis, acute trauma, or malignancy), previous knee replacement, being on the waiting list for knee surgery, physiotherapy for knee problems within the previous three months, or intra-articular injection to the knee in the previous six months.

We used two methods of recruitment: direct referral from general practitioners and retrospective review of records. We asked general practitioners, during a consultation for knee pain and aided by a prompt appearing on their computer screen when a knee pain related Read code was entered, to explain the trial to potential participants and give them a study information leaflet. After giving written consent for further contact, 


\section{Research}

participants were registered with the research centre by fax and telephoned by the study nurse to arrange a home visit. For the retrospective review of records, we did a monthly audit of each practice's computerised records to identify potential participants not recruited by the direct referral method. One general practitioner from each practice screened lists of potential participants, identified by Read codes, to identify those considered to be eligible. We posted an introductory letter and a study information leaflet to these patients, inviting them to return written consent for the study nurse to telephone them to arrange a home visit. For both methods of recruitment, the study nurse arranged a home visit within 10 working days of registration to gain written informed consent to randomisation and do a baseline assessment.

\section{Procedures}

We used a computerised random number generator to produce a pre-determined random allocation sequence, in blocks of six by general practice. We assigned each participant a unique study number, which corresponded with that on a sealed opaque envelope that contained information about participants' allocated treatment and was issued to the participant by the study nurse. To maintain blinding of the nurse, participants were instructed not to open the envelope in her presence. Follow-up was at 3, 6, and 12 months by postal questionnaire. Study nurses and researchers who collected, entered, and analysed data were unaware of treatment allocation. By necessity, participants and the health professionals delivering the interventions were not blind to allocation. We randomly assigned participants to enhanced pharmacy review, community physiotherapy, or standard advice and information reinforced by one telephone call.

\section{Interventions}

We gave each participant an information leaflet modelled on the Arthritis Research Campaign leaflet on knee osteoarthritis (www.arc.org.uk). Key messages included that knee pain is common and does not usually lead to severe disability; that individual patients can do a lot to help themselves; and that it is important to stay active and to pace activities throughout the day, to set realistic goals, and to maintain mobility in the knee joint. The leaflet provided advice about pain control and simple exercises. In addition, general practitioners were able to provide advice on analgesia to all patients. Each of the following interventions was delivered according to a written study protocol.

Enhanced pharmacy review-The aims of this intervention were to optimise pharmacological pain control and to reinforce self help messages contained in the advice leaflet. An experienced community pharmacist (MP) provided this service in general practice surgeries with access to patients' medical records; it was modelled on the "dependent prescriber" role outlined in the

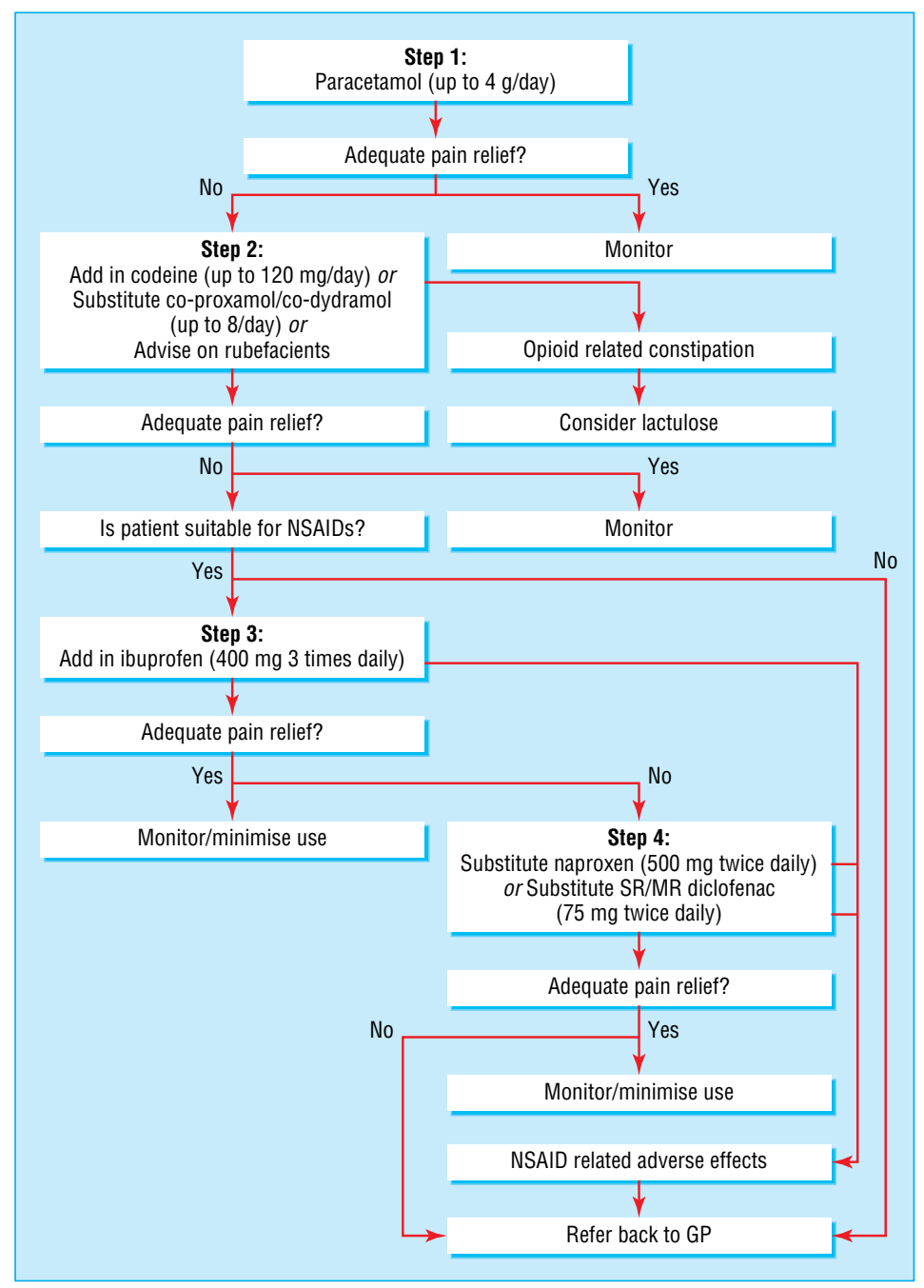

Fig 1 Pharmacy algorithm. GP=general practitioner; NSAID=non-steroidal anti-inflammatory drug; SR/MR=sustained/modified release 
Crown Report. ${ }^{7}$ MP used a pre-defined set of questions to do an initial assessment of the participants' pain control and drugs. He used standard risk factors to assess the participant's risk of adverse events from non-steroidal anti-inflammatory drugs. $\mathrm{He}$ changed participants' drugs according to a pre-defined algorithm (fig 1), taking into account their preferences, adherence, and potential drug interactions. The protocol permitted three to six sessions of approximately 20 minutes' duration over a 10 week period. In follow-up visits, MP monitored the effectiveness and acceptability of drugs and recommended changes as necessary. He recorded all treatment in a standardised format.

Community physiotherapy-The aim of this intervention was to encourage patients to engage in an active approach to managing knee pain through education about the safety and importance of exercise, pacing, pain relief, and coping strategies and an individualised exercise programme. Nineteen experienced musculoskeletal community physiotherapists delivered the intervention. They selected exercises from an agreed list from the computer software package PhysioTools (www.physiotools.net), including general aerobic exercise and specific muscle strengthening exercises (non-weight bearing and weight bearing) and stretching exercises to be done during treatment sessions and at home. The therapists increased the intensity of exercise in follow-up visits. The protocol permitted three to six sessions of approximately 20 minutes' duration over a 10 week period. Hydrotherapy, group based sessions, acupuncture, and intraarticular injections were not permitted. Therapists recorded all treatment in a standardised format.
Control intervention (information and advice leaflet)Participants in the control group received the same advice and information leaflet as the other groups. It was reinforced by one telephone call from a rheumatology nurse within seven days of randomisation.

The primary outcome measures were change at 3,6 , and 12 months after randomisation in the pain and physical function subscale scores of the Western Ontario and McMaster Universities osteoarthritis index (WOMAC). ${ }^{8}$ The psychometric properties of the WOMAC have been extensively studied in populations with knee pain in both clinical trials and postal surveys. ${ }^{9-15}$ Secondary outcome measures included participants' global assessment of change compared with baseline (five point ordinal scale), severity of pain over the previous seven days (0-10 numerical rating scale), severity rating of patient nominated main functional problem ${ }^{16}$ over the previous three days (0-10 numerical rating scale), participants' self efficacy (arthritis self-efficacy scale ${ }^{17}$ ), and psychological distress (hospital anxiety and depression scale ${ }^{18}$ ). At each follow-up point, we recorded participants' perceptions about the usefulness of their treatment (simple categorical data). We also recorded side effects of treatment, adverse events, and use of co-interventions (self reported consultation with the general practitioner or other health professional for knee pain; drug use).

We applied the guidelines suggested by the Outcome Measures in Rheumatology-Osteoarthritis Research Society International (OMERACT-OARSI) initiative for defining clinically significant responder criteria ${ }^{19} 20$ to the relevant data (WOMAC pain and function scores and participants' global assessment of

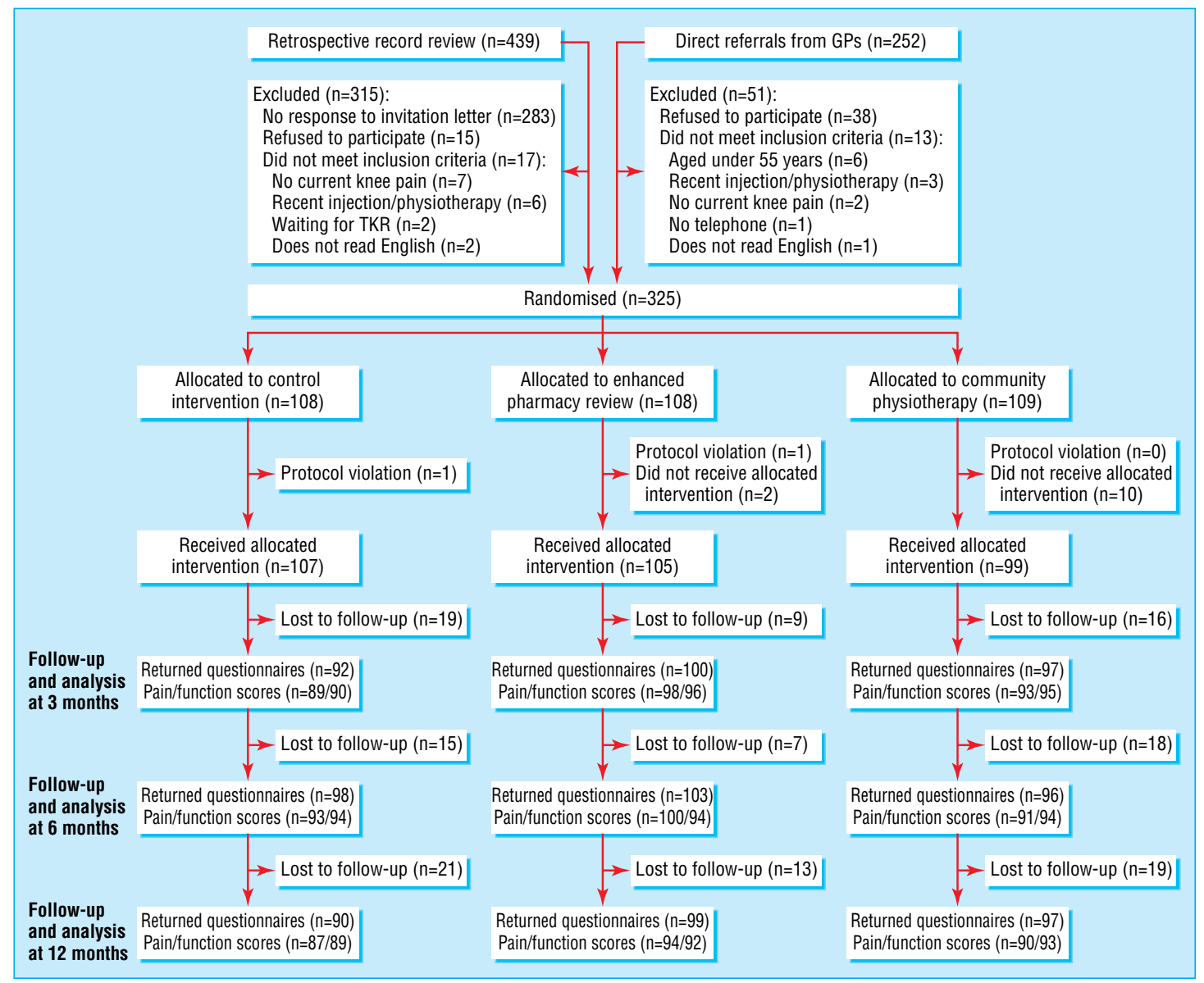

Fig 2 Trial profile. GP=general practitioner; TKR=total knee replacement 
Table 1 Baseline characteristics of participants, by treatment group. Values are numbers (percentages) unless stated otherwise

\begin{tabular}{|c|c|c|c|}
\hline Characteristics & $\begin{array}{c}\text { Control } \\
\text { intervention } \\
(n=108)\end{array}$ & $\begin{array}{c}\text { Enhanced } \\
\text { pharmacy } \\
\text { review }(n=108)\end{array}$ & $\begin{array}{c}\text { Community } \\
\text { physiotherapy } \\
(\mathrm{n}=109)\end{array}$ \\
\hline \multicolumn{4}{|l|}{ Demography } \\
\hline Mean (SD) age (years) & $68.2(8.0)$ & $67.9(8.2)$ & $67.9(8.5)$ \\
\hline Female & $70(65)$ & $68(63)$ & $71(65)$ \\
\hline Body mass index: & $(\mathrm{n}=106)$ & $(\mathrm{n}=106)$ & $(\mathrm{n}=108)$ \\
\hline Underweight/normal $(<25.0)$ & $20(19)$ & $23(22)$ & $27(25)$ \\
\hline Overweight (25.0-29.9) & $43(41)$ & $51(48)$ & $53(49)$ \\
\hline Obese $(>29.9)$ & $43(41)$ & $32(30)$ & $28(26)$ \\
\hline Socioeconomic classification: & $(\mathrm{n}=105)$ & $(n=105)$ & $(\mathrm{n}=107)$ \\
\hline Higher managerial/professional & $3(3)$ & $6(6)$ & $7(7)$ \\
\hline Lower managerial/professional & $16(15)$ & $12(11)$ & $15(14)$ \\
\hline Intermediate occupations & $11(10)$ & $18(17)$ & $16(15)$ \\
\hline Self employed & $10(10)$ & $14(13)$ & $11(10)$ \\
\hline Lower supervisory/technical & $7(7)$ & $12(11)$ & $4(7)$ \\
\hline Semiroutine occupations & $27(26)$ & $20(19)$ & $25(23)$ \\
\hline Routine occupations & $31(30)$ & $23(22)$ & $29(27)$ \\
\hline Currently employed & $20(19)$ & $23(21)$ & $22(20)$ \\
\hline \multicolumn{4}{|l|}{ Knee pain and function } \\
\hline Mean (SD) WOMAC pain score $(0-20)$ & $9.2(3.3)$ & $9.1(3.5)$ & $9.1(4.1)$ \\
\hline $\begin{array}{l}\text { Mean (SD) WOMAC function score } \\
(0-68)\end{array}$ & $30.6(12.0)$ & $29.2(12.1)$ & $30.0(14.1)$ \\
\hline Duration of pain $>3$ months & $91(84)$ & $78(72)$ & $85(78)$ \\
\hline $\begin{array}{l}\text { Mean (SD) pain severity in previous } 7 \\
\text { days (NRS) }\end{array}$ & $6.0(2.3)$ & $6.0(2.2)$ & $5.8(2.3)$ \\
\hline $\begin{array}{l}\text { Mean (SD) severity of main problem } \\
\text { (NRS) }\end{array}$ & $6.1(2.1)$ & $6.0(2.3)$ & $6.3(2.4)$ \\
\hline Mean (SD) ASE, pain (5-50) & $24.8(8.8)$ & $26.1(10.1)$ & $25.6(9.6)$ \\
\hline Mean (SD) ASE, other symptoms (6-60) & $34.1(12.3)$ & $34.3(12.2)$ & $34.0(12.3)$ \\
\hline Knee injury in previous 6 months & 9/107 (8) & $10(9)$ & $14(13)$ \\
\hline \multicolumn{4}{|l|}{ Used drugs: } \\
\hline Non-steroidal anti-inflammatory drugs & $37(34)$ & $39(36)$ & $40(37)$ \\
\hline Analgesics & $55(51)$ & $69(64)$ & $56(51)$ \\
\hline
\end{tabular}

ASE=arthritis self-efficacy scale; NRS=numerical rating scale (0-10); WOMAC=Western Ontario and McMaster Universities osteoarthritis index.

change) collected at follow-ups to define a group of "responders."

\section{Statistical analysis}

We used the pain and function subscales of the WOMAC, at six months, for the power calculation. We based this on expected changes in pain and physical function scores of $20 \%$ between the experimental treatments and the control group, assuming that pain and physical function scores may improve by $5 \%$ in the control group. ${ }^{21}$ On the basis of previously published data, ${ }^{12}{ }^{13} \mathrm{a}$ minimum of 270 participants with post-randomisation outcome data at six months would be sufficient to detect these effects with $80 \%$ power and at a $5 \%$ significance level (two tailed). We therefore recruited a total of 325 participants into the study to allow for a $20 \%$ loss to follow-up at six months.

Analysis was by intention to treat. We calculated estimates of the treatment effects (control intervention minus active treatment group) with 95\% confidence intervals and used $t$ tests for numerical data, $\chi^{2}$ tests for nominal data, and $\chi^{2}$ test for trend for ordinal data for the primary and secondary outcome measures. We did two exploratory sensitivity analyses of the mean WOMAC scores. Firstly, we did analysis of covariance by using multiple linear regression with adjustment for covariates, selected according to random differences in baseline characteristics. Secondly, we did an on-treatment analysis by restricting the comparison to participants who received their allocated treatment per protocol (defined as at least one session with either a physiotherapist or pharmacist). We assessed external validity in three ways. We compared the demographic characteristics of patients obtained through direct referral from general practitioners who were not randomised in the trial with those of trial participants. Within trial participants, we made comparisons of recruitment characteristics and treatment allocation across high and low recruiting practices and participants recruited through direct referrals and review of records.

We used Stata version 7.0 for statistical analyses. We set statistical significance at the 5\% level (two tailed). An independent steering and data monitoring committee monitored the trial. We did no interim analyses during the study period.

\section{Results}

General practitioners directly referred 252 patients, of whom we randomised 201 (80\%) (fig 2); 13 (5\%) were not eligible, and 38 $(15 \%)$ did not consent. The age and sex of these last 51 patients was similar to those randomised to the trial (mean age 67 years, $65 \%$ female). In addition, we sent letters of invitation to 439 patients after the review of records: 156 responded, of whom 124 $(79 \%)$ were randomised, $17(11 \%)$ were not eligible, and $15(10 \%)$ did not consent. Treatment allocation and baseline characteristics were similar between participants recruited directly and those recruited by record review, although the second group were less likely to report knee pain of less than three months' duration.

We randomised 325 participants to the trial: 108 to pharmacy, 109 to physiotherapy, and 108 to control. Numbers of randomised participants ranged from 1 to 88 patients per general practice. Table 1 shows the baseline characteristics of randomised participants (mean age 68 (range 55-92) years, 64\% female). Recruitment characteristics were similar between treatment groups. Treatment allocation and recruitment characteristics were similar between the highest (31 or more patients recruited) and lowest (up to 30 patients recruited) recruiting practices, although patients in the lower recruiting practices were more likely to have less than three months' duration of symptoms and had higher average WOMAC pain and physical function scores. Response to follow-up questionnaires at six months was $91 \%(\mathrm{n}=98)$ for the control group, $95 \%$ (103) for the pharmacy group, and 88\% (96) for the physiotherapy group. Those lost to follow-up at six months were more likely to be male (39\% (11/28) v 35\% (105/297)), be older (mean age 69.9 v 67.9 years), and have higher baseline WOMAC pain and function scores (pain $9.75 v 9.08$; function $32.8 v 29.7$ ) than those who completed follow-up. Concealment of treatment from the study nurse was effective: treatment allocation was revealed to the nurse by 15 of 325 participants (seven in the control intervention and four in each treatment arm).

Treatments were in line with the study protocols, and no serious adverse events were reported. In the control arm, 103 participants were contacted by telephone and one protocol violation occurred. In the pharmacy arm, 105 attended for their intervention, of whom $101(96 \%)$ had three or more intervention sessions (median 3, range 1-5), and one protocol violation occurred. A 70\% increase in the prescribing of simple and compound analgesics occurred and a $52 \%$ reduction in the prescribing of non-steroidal anti-inflammatory drugs; 104 (99\%) participants received advice reinforcing the advice leaflet. Ninety nine of 109 participants randomised to physiotherapy attended for at least one session; $83(84 \%)$ had three or more sessions (median 4, range 1-6), 97 received a home exercise programme, and 92 received advice and information. Smaller numbers had at least one session of pain reducing modalities. 
Research

Table 2 WOMAC pain and function scores at 3, 6, and 12 months' follow-up. Values are mean (SD) unless stated otherwise

\begin{tabular}{|c|c|c|c|c|c|c|c|}
\hline & \multirow{2}{*}{$\begin{array}{l}\text { Control } \\
\text { intervention } \\
\text { scores }\end{array}$} & \multicolumn{3}{|c|}{ Enhanced pharmacy review } & \multicolumn{3}{|c|}{ Community physiotherapy } \\
\hline & & Scores & $\begin{array}{c}\text { Mean difference } \\
(95 \% \mathrm{Cl})^{*}\end{array}$ & $P$ valuet & Scores & $\begin{array}{c}\text { Mean difference } \\
(95 \% \mathrm{CI})^{*}\end{array}$ & $P$ value \\
\hline \multicolumn{8}{|l|}{ WOMAC pain } \\
\hline 3 months: & $(\mathrm{n}=89)$ & $(n=98)$ & & & $(n=93)$ & & \\
\hline Absolute score & $8.99(3.7)$ & $7.49(4.0)$ & & & $7.36(4.3)$ & & \\
\hline Crude change scoreł & $0.41(2.8)$ & $1.59(3.2)$ & $1.18(0.3$ to 2.1$)$ & \multirow{2}{*}{0.006} & $1.56(3.4)$ & 1.15 (0.2 to 2.1$)$ & \multirow{2}{*}{0.008} \\
\hline Adjusted change score§ & & & $1.18(0.3$ to 2.0$)$ & & & 1.19 (0.3 to 2.1) & \\
\hline 6 months: & $(n=93)$ & $(\mathrm{n}=100)$ & & & $(\mathrm{n}=91)$ & & \\
\hline Absolute score & $8.36(3.9)$ & $7.59(4.1)$ & & & $7.51(4.8)$ & & \\
\hline Crude change score $\neq$ & $1.05(3.4)$ & $1.46(3.5)$ & $0.41(-0.6$ to 1.4$)$ & \multirow{2}{*}{0.4} & $1.19(3.9)$ & $0.14(-0.9$ to 1.2$)$ & \multirow{2}{*}{0.7} \\
\hline Adjusted change score§ & & & $0.36(-0.6$ to 1.3$)$ & & & $0.23(-0.8$ to 1.2$)$ & \\
\hline 12 months: & $(\mathrm{n}=87)$ & $(n=94)$ & & & $(n=90)$ & & \\
\hline Absolute score & $8.49(4.5)$ & $7.60(4.5)$ & & & $7.41(4.4)$ & & \\
\hline Crude change score $\neq$ & $0.74(4.1)$ & $1.37(3.9)$ & $0.63(-0.5$ to 1.8$)$ & \multirow{2}{*}{0.3} & $1.19(4.2)$ & $0.45(-0.8$ to 1.7$)$ & \multirow{2}{*}{0.3} \\
\hline Adjusted change score§ & & & $0.55(-0.6$ to 1.7$)$ & & & $0.59(-0.5$ to 1.7$)$ & \\
\hline \multicolumn{8}{|l|}{ WOMAC function } \\
\hline 3 months: & $(n=90)$ & $(n=96)$ & & & $(n=95)$ & & \\
\hline Absolute score & $30.18(12.8)$ & $25.73(13.4)$ & & & $24.27(15.2)$ & & \\
\hline Crude change score $\neq$ & $0.80(8.5)$ & $2.61(9.8)$ & $1.80(-0.8$ to 4.5$)$ & \multirow{2}{*}{0.1} & $4.79(10.8)$ & 3.99 (1.2 to 6.8$)$ & \multirow{2}{*}{0.008} \\
\hline Adjusted change score§ & & & $2.12(-0.5$ to 4.8$)$ & & & 3.65 (1.0 to 6.3$)$ & \\
\hline 6 months: & $(n=94)$ & $(n=94)$ & & & $(\mathrm{n}=94)$ & & \\
\hline Absolute score & $28.15(13.2)$ & $26.82(13.4)$ & & & $25.49(16.3)$ & & \\
\hline Crude change score $\ddagger$ & $2.74(10.5)$ & $1.52(11.4)$ & $-1.23(-4.4$ to 1.9$)$ & \multirow{2}{*}{0.5} & $3.34(12.2)$ & $0.59(-2.7$ to 3.9$)$ & \multirow{2}{*}{0.7} \\
\hline Adjusted change score§ & & & $-0.96(-4.0$ to 2.1$)$ & & & $0.66(-2.5$ to 3.8$)$ & \\
\hline 12 months: & $(n=89)$ & $(n=92)$ & & & $(n=93)$ & & \\
\hline Absolute score & $28.95(14.4)$ & $27.14(14.6)$ & & & $24.83(15.3)$ & & \\
\hline Crude change score $\neq$ & $1.65(12.3)$ & $1.15(11.7)$ & $-0.49(-4.0$ to 3.0$)$ & \multirow{2}{*}{0.8} & $4.00(13.2)$ & $2.35(-1.4$ to 6.1$)$ & \multirow{2}{*}{0.2} \\
\hline Adjusted change score§ & & & -0.39 (-3.8 to 3.0$)$ & & & $2.41(-1.1$ to 5.9$)$ & \\
\hline
\end{tabular}

WOMAC=Western Ontario and McMaster Universities osteoarthritis index.

${ }^{*}$ Difference in mean scores (control - active treatment).

†Derived from adjusted regression analysis.

$\ddagger$ Change in score from baseline.

§Mean difference adjusted for age, sex, baseline WOMAC pain/function score, and baseline duration of pain.

\section{Primary outcome}

At three months, significant improvements in WOMAC pain and function scores occurred in the physiotherapy group, and in pain scores in the pharmacy group, when we compared each intervention separately with control. The significant differences persisted after adjustment for sex, age, and baseline WOMAC scores and duration of pain.

No statistically significant differences existed in mean WOMAC change scores between the control group and the pharmacy or physiotherapy groups at six and 12 months (table 2). Figure 3 illustrates the changes in the WOMAC pain and function scores during the whole follow-up period. We found similar results when we did the sensitivity analysis.

\section{Secondary outcomes}

Table 3 shows participants' perceived global assessment of change in their knee problem compared with baseline. More of the pharmacy and physiotherapy groups, compared with the control group, were classified as responders according to the OMERACT-OARSI criteria at each of the three follow-up points, but the difference was statistically significant only at three months. Table 4 shows data on other secondary outcome measures.

\section{Co-interventions}

A higher proportion of participants in the control group than in the physiotherapy group reported consulting their general practitioner for knee pain during the six month follow-up (table 5). Self reported use of non-steroidal anti-inflammatory drugs and simple analgesia in the six month post-randomisation period was significantly lower in the physiotherapy group than in the control group $(-15 \%, 95 \%$ confidence interval $-2 \%$ to $-28 \%$; and $-16 \%,-3 \%$ to $-29 \%)$. In the pharmacy group, use of nonsteroidal anti-inflammatory drugs was significantly lower than

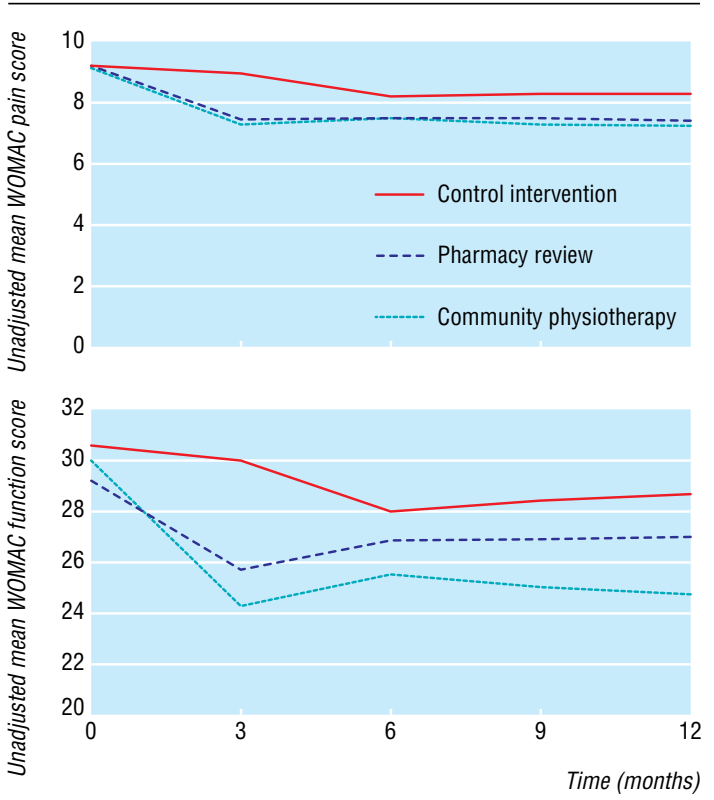

Fig 3 Mean Western Ontario and McMaster Universities osteoarthritis index (WOMAC) scores at recruitment and at 3, 6, and 12 months' follow-up. Top: WOMAC pain scores. Bottom: WOMAC function scores 
Table 3 Global assessment of overall change and OMERACT-OARSI response*. Values are numbers (percentages)

\begin{tabular}{|c|c|c|c|c|c|}
\hline & \multirow{2}{*}{$\begin{array}{l}\text { Control } \\
\text { intervention } \\
\text { global } \\
\text { assessment }\end{array}$} & \multicolumn{2}{|c|}{$\begin{array}{c}\text { Enhanced pharmacy } \\
\text { review }\end{array}$} & \multicolumn{2}{|c|}{$\begin{array}{l}\text { Community } \\
\text { physiotherapy }\end{array}$} \\
\hline & & $\begin{array}{c}\text { Global } \\
\text { assessment }\end{array}$ & $\begin{array}{c}P \\
\text { value }\end{array}$ & $\begin{array}{c}\text { Global } \\
\text { assessment }\end{array}$ & $\begin{array}{c}P \\
\text { value† }\end{array}$ \\
\hline \multicolumn{6}{|l|}{ Global assessment } \\
\hline 3 months: & $(\mathrm{n}=91)$ & $(\mathrm{n}=98)$ & & $(\mathrm{n}=94)$ & \\
\hline Much better & $7(8)$ & $19(19)$ & \multirow{5}{*}{0.0002} & $19(20)$ & \multirow{5}{*}{$<0.0001$} \\
\hline Better & $19(21)$ & $31(32)$ & & $31(33)$ & \\
\hline Same & $42(46)$ & $36(37)$ & & $37(39)$ & \\
\hline Worse & $18(20)$ & $12(12)$ & & $7(7)$ & \\
\hline Much worse & $5(6)$ & 0 & & 0 & \\
\hline 6 months: & $(n=93)$ & $(n=99)$ & & $(\mathrm{n}=91)$ & \\
\hline Much better & $13(14)$ & $10(10)$ & \multirow{5}{*}{0.03} & $18(20)$ & \multirow{5}{*}{0.09} \\
\hline Better & $15(16)$ & $37(37)$ & & $26(29)$ & \\
\hline Same & $46(49)$ & $44(44)$ & & $31(34)$ & \\
\hline Worse & $17(18)$ & $8(8)$ & & $13(14)$ & \\
\hline Much worse & $2(2)$ & 0 & & $3(3)$ & \\
\hline 12 months: & $(\mathrm{n}=89)$ & $(n=94)$ & & $(n=94)$ & \\
\hline Much better & $11(13)$ & $13(14)$ & \multirow{5}{*}{0.2} & $15(16)$ & \multirow{5}{*}{0.2} \\
\hline Better & $11(13)$ & $19(20)$ & & $13(13)$ & \\
\hline Same & $39(43)$ & $37(39)$ & & $44(47)$ & \\
\hline Worse & $22(25)$ & $21(22)$ & & $20(21)$ & \\
\hline Much worse & $6(7)$ & $4(4)$ & & $2(2)$ & \\
\hline \multicolumn{6}{|c|}{ OMERACT-OARSI response } \\
\hline \multicolumn{6}{|c|}{3 months: } \\
\hline High/improvement & $6 / 11$ & $18 / 14$ & \multirow{2}{*}{0.04} & $19 / 18$ & \multirow{2}{*}{0.003} \\
\hline Total response & 17/89 (19) & 32/97 (33) & & $37 / 93(40)$ & \\
\hline \multicolumn{6}{|l|}{6 months: } \\
\hline High/improvement & $8 / 16$ & $17 / 18$ & \multirow{2}{*}{0.2} & $23 / 11$ & \multirow{2}{*}{0.1} \\
\hline Total response & $24 / 92(26)$ & $35 / 100(35)$ & & $34 / 92$ (37) & \\
\hline \multicolumn{6}{|l|}{12 months: } \\
\hline High/improvement & $13 / 11$ & $15 / 10$ & \multirow{2}{*}{0.8} & $21 / 11$ & \multirow{2}{*}{0.3} \\
\hline Total response & $24 / 86$ (28) & $25 / 93$ (27) & & 32/89 (36) & \\
\hline
\end{tabular}

*Criteria suggested by the Outcome Measures in Rheumatology-0steoarthritis Research Society International (OMERACT-OARSI) initiative for defining clinically significant response. ${ }^{19}$

$\dagger \chi^{2}$ test for trend for global assessment and $\chi^{2}$ test for OMERACT-OARSI response.

for controls $(-16 \%,-3 \%$ to $-29 \%)$, but use of simple analgesia was significantly higher $(15 \%, 0 \%$ to $28 \%)$.

\section{Discussion}

Our findings support the feasibility, acceptability, and short term clinical effectiveness of community physiotherapy and enhanced pharmacy review in the management of people aged over 55 with knee pain. Statistically significant improvements in pain scores occurred in participants allocated to enhanced pharmacy review or community physiotherapy and in function scores in those allocated to physiotherapy at three months compared with controls. These differences were not sustained to six or 12 months. To evaluate the clinical significance of the size of the differences, we applied the response criteria suggested by the OMERACT-OARSI group. ${ }^{19}{ }^{20}$ According to these rather stringent criteria, a substantially and statistically significant higher proportion of the physiotherapy group $(40 \%)$ and pharmacy group (33\%) than the control group (19\%) were classified as responders at three months.

\section{Effects on drug use and general practice consultations}

One consistent finding, with important clinical implications, was that prescribing of non-steroidal anti-inflammatory drugs was reduced in both pharmacy and physiotherapy groups compared with control. At six months, use of non-steroidal antiinflammatory drugs was $16 \%$ lower in the pharmacy group and
$15 \%$ lower in the physiotherapy group than in the control group, with no increase in reporting of pain and high levels of patient satisfaction. This has important safety implications. Nonsteroidal anti-inflammatory drugs are the most common cause of iatrogenic disease and are not recommended for long term use, particularly in elderly people, in whom the risk of complications is high. Recalled consultation with general practitioners for knee pain was significantly lower in the six month period after the physiotherapy intervention than after the control intervention.

\section{Strengths of the trial}

Importantly, this pragmatic study evaluated two approaches to delivering evidence based care for patients with knee pain (pharmacy and physiotherapy)-it did not investigate the efficacy of specific modalities (tablets and exercise). Our trial had high internal validity, shown by adequate recruitment, concealed randomisation, high follow-up rates, and effective blinding of the research team. Experienced practitioners delivered the interventions, in accordance with standardised study protocols designed to reflect evidence based practice while retaining sufficient flexibility to ensure that the therapists could develop individualised treatment plans to reflect clinical need. We deliberately chose not to restrict our trial to people with radiographically diagnosed osteoarthritis in order to reflect current clinical practice, in which treatment choices for people with knee pain seeking health care are made on the basis of presenting symptoms rather than radiographic changes.

Recruitment rate varied considerably across general practices; a single practice recruited a quarter of the study population. We found no evidence that this adversely affected the external validity of the trial or diminished the generalisability of the "usual" care given in the trial. For example, baseline use of non-steroidal anti-inflammatory drugs (a proxy measure of general practitioners' behaviour) was similar in high and low recruiting practices.

\section{Limitations}

One potential weakness of our trial is the lack of information about patients' adherence to treatment, which is likely to be an important determinant of clinical outcome. ${ }^{22}{ }^{23}$ We measured adherence in a limited fashion by the number of sessions attended rather than the actual level of ongoing participation in, for example, home exercises in the physiotherapy group or numbers of tablets taken in the pharmacy group. Adherence may have decreased over time, as has been shown in other studies, $^{22}$ and this may be one explanation for the lack of a long term superior clinical effect of the pharmacy and physiotherapy interventions over control in our trial. A further explanation may lie in the "dosage" of our interventions. The interventions were based on recommendations from international guidelines for the management of osteoarthritis of the knee. ${ }^{24} 25$ The exact content was drawn up in collaboration with general practitioners, pharmacists, and physiotherapists to reflect current UK primary care practice and was agreed with the treating clinicians in two workshops before the trial began. Although the protocols permitted up to six sessions with a physiotherapist or pharmacist, the interventions were actually delivered in fewer sessions (equating to a median of 53 minutes of contact time in the pharmacy group and 80 minutes in the physiotherapy group). More intensive initial treatment ${ }^{26}$ or systematic approaches to follow-up, including "top-up" treatments or open access to practitioners to manage flares of symptoms, might have improved long term outcomes. 
Research

Table 4 Secondary outcome measures at 3, 6, and 12 months' follow-up, by treatment

\begin{tabular}{|c|c|c|c|c|c|c|c|}
\hline \multirow[b]{2}{*}{ End point } & \multirow{2}{*}{$\begin{array}{l}\text { Control intervention } \\
\text { measurement }\end{array}$} & \multicolumn{3}{|c|}{ Enhanced pharmacy review } & \multicolumn{3}{|c|}{ Community physiotherapy } \\
\hline & & Measurement & Difference* $(95 \% \mathrm{CI})$ & $P$ value & Measurement & Difference $^{*}(95 \% \mathrm{CI})$ & P value \\
\hline \multicolumn{8}{|c|}{ Knee pain and function (mean (SD)) } \\
\hline \multicolumn{8}{|c|}{ Change in pain severity $\ddagger$ (numerical rating scale): } \\
\hline 3 months & $0.54(2.2)$ & $1.34(2.5)$ & $-0.72(-1.4$ to -0.1$)$ & 0.04 & $1.40(2.3)$ & $-0.84(-1.5$ to -0.2$)$ & 0.01 \\
\hline 6 months & $0.84(2.5)$ & $1.37(2.4)$ & $-0.41(-1.1$ to 0.3$)$ & 0.3 & $1.22(2.4)$ & $-0.34(-1.1$ to 0.4$)$ & 0.4 \\
\hline 12 months & $0.80(2.8)$ & $1.25(2.8)$ & $-0.32(-1.2$ to 0.5$)$ & 0.5 & $0.58(2.8)$ & $0.01(-0.8$ to 0.9$)$ & 0.9 \\
\hline \multicolumn{8}{|c|}{ Change in severity of main problem $\ddagger$ (numerical rating scale): } \\
\hline 3 months & $0.03(2.2)$ & $0.50(2.5)$ & $-0.46(-1.2$ to 0.3$)$ & 0.2 & $1.20(2.7)$ & $-1.06(-1.8$ to -0.3$)$ & 0.005 \\
\hline 6 months & $0.00(2.3)$ & $0.48(2.5)$ & -0.39 (-1.1 to 0.3$)$ & 0.3 & $1.31(3.0)$ & $-1.22(-2.0$ to -0.4$)$ & 0.002 \\
\hline 12 months & $0.29(2.7)$ & $0.34(3.1)$ & $-0.01(-0.9$ to 0.9$)$ & 0.9 & $0.70(3.3)$ & $-0.40(-1.3$ to 0.5$)$ & 0.4 \\
\hline \multicolumn{8}{|c|}{ Psychological measures (mean (SD)) } \\
\hline \multicolumn{8}{|c|}{ Change in arthritis self-efficacy scale, painł: } \\
\hline 3 months & $-1.87(9.9)$ & $-1.16(9.5)$ & -0.88 (-3.8 to 2.0$)$ & 0.6 & $-4.62(9.5)$ & $2.67(-0.3$ to 5.6$)$ & 0.07 \\
\hline 6 months & $-1.91(9.1)$ & $-1.25(9.6)$ & $-1.08(-3.9$ to 1.7$)$ & 0.4 & $-3.24(12.5)$ & $1.29(-2.1$ to 4.6$)$ & 0.5 \\
\hline 12 months & $-3.20(10.1)$ & $0.38(10.5)$ & $-3.58(-6.7$ to -0.4$)$ & 0.03 & $-4.38(12.7)$ & $1.31(-2.3$ to 4.9$)$ & 0.5 \\
\hline \multicolumn{8}{|c|}{ Change in arthritis self-efficacy scale, other symptomsł: } \\
\hline 3 months & $-1.47(12.3)$ & $-2.03(9.9)$ & $0.53(-2.8$ to 3.8$)$ & 0.8 & $-4.33(10.3)$ & $2.55(-0.7$ to 5.8$)$ & 0.1 \\
\hline 6 months & $-2.15(10.4)$ & $-1.87(10.2)$ & $-1.30(-4.3$ to 1.7$)$ & 0.4 & $-3.42(13.4)$ & $1.30(-2.3$ to 4.9$)$ & 0.5 \\
\hline 12 months & $-3.31(12.6)$ & $-0.24(12.7)$ & $-3.44(-7.3$ to 0.5$)$ & 0.08 & $-4.49(13.7)$ & 1.48 (-2.5 to 5.4$)$ & 0.5 \\
\hline \multicolumn{8}{|c|}{ Change in hospital anxiety and depression scale, depression‡: } \\
\hline 3 months & $-0.28 \quad(2.0)$ & $0.22(2.2)$ & $-0.55(-1.2$ to 0.1$)$ & 0.08 & $0.14(2.0)$ & $-0.40(-1.0$ to 0.2$)$ & 0.2 \\
\hline 6 months & $-0.32(2.3)$ & $0.18(2.2)$ & -0.46 (-1.1 to 0.2$)$ & 0.2 & $0.10(2.8)$ & $-0.37(-1.1$ to 0.4$)$ & 0.3 \\
\hline 12 months & $-0.24(2.2)$ & $-0.27(2.1)$ & $0.01(-0.7$ to 0.7$)$ & 0.9 & $0.02(2.8)$ & $-0.27 \quad(-1.1$ to 0.5$)$ & 0.5 \\
\hline \multicolumn{8}{|c|}{ Change in hospital anxiety and depression scale, anxietył: } \\
\hline 3 months & $-0.17(2.5)$ & $0.22(2.6)$ & $-0.46(-1.2$ to 0.3$)$ & 0.2 & $0.59(2.6)$ & $-0.66(-1.4$ to 0.01$)$ & 0.09 \\
\hline 6 months & $0.24(2.5)$ & $0.15(2.6)$ & $0.10(-0.6$ to 0.8$)$ & 0.8 & $0.81(3.1)$ & $-0.53(-1.4$ to 0.3$)$ & 0.2 \\
\hline 12 months & $-0.28 \quad(2.8)$ & $0.05(2.8)$ & $-0.23(-1.1$ to 0.6$)$ & 0.6 & $0.50(2.9)$ & -0.69 ( -1.5 to 0.2$)$ & 0.1 \\
\hline \multicolumn{8}{|c|}{ Treatment usefulness and satisfaction (№ (\%)) } \\
\hline \multicolumn{8}{|c|}{ Treatment useful for reducing knee pain: } \\
\hline 3 months & $27 / 89(30)$ & $45 / 97(46)$ & $-16 \%(-29$ to -2$)$ & 0.02 & $57 / 90(63)$ & $-33 \%(-46$ to -19$)$ & $<0.0001$ \\
\hline 6 months & $22 / 89(23)$ & $37 / 93(40)$ & $-17 \%(-29$ to -3$)$ & 0.02 & $47 / 87(54)$ & $-31 \%(-43$ to -16$)$ & $<0.0001$ \\
\hline 12 months & $19 / 82(23)$ & $36 / 91(40)$ & $-17 \%(-30$ to -3$)$ & 0.02 & $43 / 93(46)$ & $-23 \% \quad(-36$ to -9$)$ & 0.001 \\
\hline \multicolumn{8}{|c|}{ Treatment useful for helping to return to usual activities: } \\
\hline 3 months & $20 / 87(23)$ & $38 / 96(40)$ & $-17 \%(-29$ to -3$)$ & 0.01 & $45 / 90(50)$ & $-27 \% \quad(-40$ to -13$)$ & $<0.0001$ \\
\hline 6 months & $21 / 89(24)$ & $30 / 94(32)$ & $-8 \% \quad(-21$ to 5$)$ & 0.2 & $40 / 87(46)$ & $-22 \%(-35$ to -8$)$ & 0.002 \\
\hline 12 months & $18 / 78 \quad(23)$ & $29 / 91 \quad(32)$ & $-9 \% \quad(-22$ to 4$)$ & 0.2 & $35 / 90(39)$ & $-16 \%(-29$ to -2$)$ & 0.03 \\
\hline \multicolumn{8}{|c|}{ Treatment useful in giving practical advice: } \\
\hline 3 months & $46 / 88(52)$ & $71 / 96(74)$ & $-22 \%(-34$ to -8$)$ & 0.002 & $77 / 90(86)$ & $-33 \%(-45$ to -20$)$ & $<0.0001$ \\
\hline 6 months & $46 / 88(52)$ & $62 / 94(66)$ & $-14 \%(-27$ to 0.1$)$ & 0.06 & $69 / 85(81)$ & $-29 \%(-41$ to -15$)$ & $<0.0001$ \\
\hline 12 months & $29 / 80(35)$ & $55 / 91(60)$ & $-24 \%(-38$ to -9$)$ & 0.002 & $59 / 90(66)$ & $-29 \% \quad(-43$ to -14$)$ & $<0.0001$ \\
\hline \multicolumn{8}{|c|}{ Satisfied with received treatment: } \\
\hline 3 months & $41 / 88(46)$ & $64 / 96(67)$ & $-20 \%(-33$ to -6$)$ & 0.006 & $67 / 90(74)$ & $-28 \%(-47$ to -14$)$ & $<0.0001$ \\
\hline 6 months & $37 / 86(42)$ & $53 / 93(57)$ & $-14 \% \quad(-28$ to 1$)$ & 0.06 & $60 / 86(70)$ & $-27 \%(-40$ to -12$)$ & $<0.0001$ \\
\hline 12 months & $27 / 82$ (33) & $47 / 91 \quad(52)$ & $-19 \%(-32$ to -4$)$ & 0.01 & $51 / 91 \quad(56)$ & $-23 \% \quad(-37$ to -8$)$ & 0.002 \\
\hline
\end{tabular}

*Difference (control - active treatment): derived from adjusted regression (age, sex, baseline WOMAC pain/function score, and baseline duration of pain) for numerical outcomes. †Derived from adjusted regression analysis for numerical outcomes and $\chi^{2}$ test for categorical outcomes.

$\ddagger$ Change in scores from baseline.

\section{Implications for practice}

Physiotherapists working in community settings are ideally placed to deliver a package of care that incorporates self help messages into an exercise based treatment programme and to shift the management of chronic musculoskeletal problems away from the general practitioner. ${ }^{27}$ Although exercise based interventions have shown beneficial effects for older adults with knee pain, effect sizes are small, at best, and are short lived. ${ }^{28}$ Similarly, community pharmacists in the United Kingdom have been linked with a new role as "supplementary prescribers," which allows them to review and, if necessary, prescribe certain drugs within an agreed clinical management plan for patients whose condition has been assessed by an independent prescriber (such as the general practitioner). Interventions by pharmacists have been shown to favourably influence prescribing to reduce adverse drug reactions, improve the appropriate- ness of drug use, reduce drug costs, and improve compliance in a range of conditions. ${ }^{29-34}$ Our trial adds to this evidence by showing that evidence based care for adults over 55 with knee pain, delivered by primary care pharmacists and physiotherapists, results in short term improvements in health outcome, reduction in use of non-steroidal anti-inflammatory drugs, and high patient satisfaction. Community physiotherapy also seemed to effect a long term shift in consultation behaviour away from the traditional general practitioner led model of care. The challenge posed by these results is to investigate how the early clinical benefits seen might be enhanced in the longer term and whether potential reductions in use of health care make these interventions cost effective as first line primary care management strategies. 
Table 5 Self reported consultations with healthcare practitioners and drug use at six month follow-up. Values are numbers (percentages) unless stated otherwise

\begin{tabular}{|c|c|c|c|c|c|c|c|}
\hline & \multirow{2}{*}{$\begin{array}{l}\text { Control intervention } \\
\text { co-interventions } \\
(\mathrm{n}=100)\end{array}$} & \multicolumn{3}{|c|}{ Enhanced pharmacy review $(n=104)$} & \multicolumn{3}{|c|}{ Community physiotherapy ( $\mathrm{n}=101)$} \\
\hline & & Co-interventions & $\begin{array}{c}\text { Difference (\%) } \\
(95 \% \mathrm{Cl})\end{array}$ & $P$ value & Co-interventions & $\begin{array}{c}\text { Difference (\%) } \\
(95 \% \mathrm{Cl})\end{array}$ & $P$ value \\
\hline \multicolumn{8}{|c|}{ Consulted with healthcare practitioner } \\
\hline General practitioner & $25(25)$ & $21(20)$ & $5(-7$ to 16$)$ & 0.4 & $10(10)$ & 15 (5 to 25$)$ & 0.005 \\
\hline Osteopath & $2(2)$ & 0 & $2(-2$ to 7$)$ & 0.2 & 0 & $2(-2$ to 7$)$ & 0.2 \\
\hline Chiropractor & $1(1)$ & 1 (1) & $0(-4$ to 5$)$ & 1.0 & 0 & $1(-3$ to 6$)$ & 0.3 \\
\hline Pharmacist $^{*}$ & $3(3)$ & 1 (1) & $2(-3$ to 8$)$ & 0.3 & 0) & $3(-1$ to 9$)$ & 0.08 \\
\hline Rheumatologist & $5(5)$ & $1(1)$ & $4(-1$ to 10$)$ & 0.09 & 1 (1) & $4(-1$ to 10$)$ & 0.1 \\
\hline${\text { Physiotherapist }{ }^{\star}}$ & $8(8)$ & $4(4)$ & $4(-3$ to 12$)$ & 0.2 & 0 & 8 (3 to 15$)$ & 0.004 \\
\hline Orthopaedic consultant & $5(5)$ & $3(3)$ & $2(-4$ to 9$)$ & 0.4 & $3(3)$ & $2(-4$ to 9$)$ & 0.5 \\
\hline Acupuncturist & $4(4)$ & 0 & 4 (0 to 10$)$ & 0.04 & 1 (1) & $3(-2$ to 9$)$ & 0.2 \\
\hline \multicolumn{8}{|l|}{ Used drugs } \\
\hline Non-steroidal anti-inflammatory drugs & $44(44)$ & $29(28)$ & 16 (3 to 29 ) & 0.02 & $29(29)$ & 15 (2 to 28$)$ & 0.02 \\
\hline Analgesics & $58(58)$ & $76(73)$ & $-15(-28$ to 0$)$ & 0.02 & $42(42)$ & 16 (3 to 29 ) & 0.02 \\
\hline
\end{tabular}

${ }^{*}$ Contacts do not include those as part of trial interventions.

We thank the following organisations and people directly involved with the study for their support: the rheumatology nurses; the community physiotherapists and physiotherapy managers; the North Staffordshire GP Research Network and all the general practitioners who referred patients to the trial; the health informatics team, administration team, Peter Croft, Rhian Hughes, and Panos Barlas from the Primary Care Musculoskeletal Research Centre; members of the independent Data Monitoring and Ethics Committee; and the members of the public who took part in the study.

Contributors: All authors contributed to interpreting the results and drafting and revising the article. The original question was proposed by a research discussion group of general practitioners, therapists, and community pharmacists from North Staffordshire convened by the North Staffordshire NHS Primary Care Consortium. EMH contributed to the design, funding application, and management of the trial. NEF contributed to the management of the trial, the design of the physiotherapy intervention, and data collection. ET contributed to the design of the trial and data analysis. GP contributed to the design and funding application of the trial. MP contributed to the design of the pharmacy intervention and data collection of the trial. HEY contributed to the recruitment procedures of the trial and data collection and management. $\mathrm{AB}$ contributed to the design of the pharmacy intervention. JS contributed to the design and funding application of the trial. EMH is the guarantor.

Funding: Arthritis Research Campaign, North Staffordshire Primary Care Research Consortium, and the Department of Health National Co-ordinating Centre for Research Capacity Development. NEF is funded

\section{What is already known on this topic}

Current guidelines for the primary care management of knee pain and osteoarthritis emphasise the importance of non-pharmacological approaches such as education and exercise

The traditional general practitioner led service to deliver evidence based interventions is increasingly unsustainable, and alternative models are needed

\section{What this study adds}

Enhanced pharmacy review and community physiotherapy resulted in short term, clinically significant improvements compared with control in people aged 55 or over with knee pain

Both interventions were associated with high patient satisfaction and resulted in a substantial reduction in use of non-steroidal anti-inflammatory drugs

Community physiotherapy effected a shift in care away from the traditional general practitioner led model by a primary care career scientist award from the Department of Health and NHS R\&D. The sponsors of the study had no role in the study design, data collection, data analysis, data interpretation, or writing of the report. Competing interests: None declared.

Ethical approval: North Staffordshire local research ethics committee.

1 Hochberg MC, Altman RD, Brandt KD, Clark BM, Dieppe PA, Griffin MR, et al. Guidelines for the medical management of osteoarthritis. Part II: osteoarthritis of the knee. Arthritis Rheum 1995;38:1541-6.

2 Lane NE, Thompson JM. Management of osteoarthritis in the primary-care setting: an evidence-based approach to treatment. Am J Med 1997;103:25-30S.

3 Altman RD, Lozada CJ. Practice guidelines in the management of osteoarthritis. Osteoarthritis Cartilage 1998;6:22-4.

4 Primary Care Rheumatology Society. The management of osteoarthritis-guidelines 1999 (available from the PCRS: www.pcrsociety.org.uk).

5 Mazzuca SA, Brandt KD, Katz BP, Dittus RS, Freund DA, Lubitz R, et al. Comparison of general internists, family physicians, and rheumatologists managing patients with symptoms of osteoarthritis of the knee. Arthritis Care Res 1997;10:289-99.

6 Department of Health. Supporting people with long term conditions: an NHS and social care model to support local innovation and integration. London: DoH, 2005. Available at www.dh.gov.uk/publications (gateway reference 4230).

7 Review Team for the Prescribing, Supply and Administration of Medicines. Review of prescribing, supply and administration of medicines: final report (Crown Report). London: prescribing, supply and administ
Department of Health, 1999.

8 Bellamy N. WOMAC osteoarthritis index: a user's guide. Ontario: London Health Services, McMaster University, 1996.

9 Hart DJ, Spector TD. The classification and assessment of osteoarthritis. Baillière's Clin Rheumatol 1995;9:407-32.

10 Sun Y, Sturmer T, Gunther KP, Brenner H. Reliability and validity of clinical outcome measurements for osteoarthritis of the hip and knee-a review of the literature. Clin Rheumatol 1997;16:185-98.

11 Bellamy N. Osteoarthritis clinical trials: candidate variables and clinimetric properties. J Rheumatol 1997;24:768-78.

12 O'Reilly SC, Muir KR, Doherty M. Effectiveness of home exercise on pain and disability from osteoarthritis of the knee: a randomised controlled trial. Ann Rheum Dis
$1999 ; 58: 15-9$.

13 Bellamy N, Kean WF, Buchanan WW, Gerecz-Simon E, Campbell J. Double blind randomised controlled trial of sodium meclofenamate (Meclomen) and diclofenac sodium (Voltaren): post validation reapplication of the WOMAC osteoarthritis index. $J$ Rheumatol 1992;19:153-9.

14 Bellamy N, Buchanan WW, Goldsmith C, Campbell J, Stitt LW. Validation of WOMAC: a health status instrument for measuring clinically important patient relevant outcomes to antirheumatic drug therapy in patients with $\mathrm{OA}$ of the hip or knee. $J$ Rheumatol 1988;15:1833-40.

15 Jinks C, Jordan K, Croft P. Measuring the population impact of knee pain and disability with the Western Ontario and McMaster Universities osteoarthritis index (WOMAC). Pain 2002;100:55-64.

16 Beurskens AJHM, de Vet HCW, Koke AJA, Regtop W, van der Heijden GJMG, Knipschild PG. Efficacy of traction for non-specific low-back pain: 5-week results of a randomised clinical trial. Lancet 1995;346:1596-600.

17 Barlow JH, Williams RB, Wright C. The arthritis self-efficacy scale in a UK context. Psychol Health Med 1997;2:5-19.

18 Zigmond AS, Snaith RP. The hospital anxiety and depression scale. Acta Psychiatr Scand 1983;67:361-70.

19 Pham T, Van der Heijde D, Lassere M, Altman RD, Anderson JJ, Bellamy N, et al. Outcome variables for osteoarthritis clinical trials: the OMERACT-OARSI set of responders criteria.J Rheumatol 2003;30:1648-54.

20 Pham T, Van der Heijde D, Altman RD, Anderson IJ, Bellamy N, Hochberg M, et al. OMERACT-OARSI initiative: Osteoarthritis Research Society International set of OMERACT-OARSI initiative: Osteoarthritis Research Society International set of
responder criteria for osteoarthritis clinical trials revisited. Osteoarthritis Cartilage responder crite

21 Weinberger M, Tierney WM, Cowper PA, Katz BP. Booher PA. Cost-effectiveness of increased telephone contact for patients with osteoarthritis: a randomised, controlled trial. Arthritis Rheum 1993;36:243-6. 
22 Van Gool CH, Penninx BWJH, Kempen GIJM, Rejeski WJ, Miller GD, van Eijk JTM, et al. Effects of exercise adherence on physical function among overweight older adults with knee osteoarthritis. Arthritis Rheum 2005;53:24-32.

23 Ettinger WH Jr, Burns R, Messier SP, Applegate W, Rejeski WJ, Morgan T, et al. The fitness arthritis and seniors trial (FAST): a randomised trial comparing aerobic exercise and resistance exercise with a health education program in older adults with knee osteoarthritis. JAMA 1997;277:25-31.

24 Pencharz JN, Grigoriadis E, Jansz GF, Bombardier C. A critical appraisal of clinical practice ouideline for the treatment of lower-limb osteoarthritis. Arthritis Res 2002;4:36-43.

25 Jordan KM, Arden NK, Doherty M, Bannwarth B, Bijlsma JW, Dieppe P, et al. EULAR recommendations 2003: an evidence based approach to the management of knee osteoarthritis: report of a task force of the Standing Committee for International Clinical Studies Including Therapeutic Trials (ESCISIT). Ann Rheum Dis 2003;62:1145-55.

26 Deyle GD, Allison SC, Matekel RL, Ryder MG, Stang JM, Gohdes DD, et al. Physical therapy treatment effectiveness for osteoarthritis of the knee: a randomised comparison of supervised clinical exercise and manual therapy procedures versus a home exercise programme. Phys Ther 2005;85:1301-17.

27 Hurley M, Dziedzic K, Bearne L, Sim J, Bury T. Evidence briefing 3: clinical and cost effectiveness of physiotherapy in the management of older people with common rheumatological conditions. London: Chartered Society of Physiotherapy, 2002. (www.csp.org.uk/uploads/ documents/evidencebrief_rheumatology_EB03.pdf).

28 Bennell K, Hinman R. Exercise as a treatment for osteoarthritis. Curr Opin Rheumatol 2005;17:634-40.

29 Haynes RB, McKibbon KA, Kanani R. Systematic review of randomised trials of interventions to assist patients to follow prescriptions for medications. Lancet 1996;348:383-6.

30 Rollason V, Vogt N. Reduction of polypharmacy in the elderly: a systematic review of the role of pharmacist. Drugs Aging 2003;20:817-32.

31 Roughead L, Semple S, Vitry A. The value of pharmacist professional services in the community setting: a systematic review of the literature 1990-2002. Quality Use of Medicines and
Pharmacy Research Centre, School of Pharmaceutical, Molecular and Biomedical Sciences, University of South Australia, Australia, 2002.

32 Fish A, Watson MC, Bond CM. Practice-based pharmaceutical services: a systematic review. Int J Pharm Pract 2002;10:225-33.

33 Tully MP, Seston EM. Impact of pharmacists providing a prescription review and monitoring service in ambulatory care or community practice. Ann Pharmacother 2000;34:1320-31.

34 Beney J, Bero LA, Bond C. Expanding roles of outpatient pharmacists: effects on health care utilisation, costs, and patient outcomes. Cochrane Database Syst Rev 2000;(2):CD000336

(Accepted 12 September 2006)

doi 10.1136/bmj.38977.590752.0B

Primary Care Musculoskeletal Research Centre, Keele University, Keele Elaine M Hay professor/consultant in community rheumatology

Nadine E Foster DoH primary care career scientist and senior lecturer in therapies

Elaine Thomas senior lecturer in biostatistics

George Peat senior lecturer in clinical epidemiology

Mike Phelan medication review pharmacist

Hannah E Yates research nurse

Julius Sim professor of health care research

Medicines Management, Keele University

Alison Blenkinsopp professor of the practice of pharmacy

Correspondence to: E M Hay e.m.hay@cphc.keele.ac.uk 